\title{
D-band Transmission Hub for Point to MultiPoint Wireless Distribution
}

\author{
Maruf Hossain ${ }^{1}$, Viktor Krozer ${ }^{2}$, Trung Le ${ }^{3}$, Rupa Basu ${ }^{4}$, Rosa Letizia ${ }^{4}$, Ernesto Limiti ${ }^{5}$, François Magne ${ }^{6}$, Marc \\ Marilier $^{7}$, Antonio Ramirez ${ }^{8}$, Jeevan M Rao ${ }^{4}$, Giacomo Ulisse ${ }^{2}$, Borja Vidal ${ }^{9}$, Hadi Yacob ${ }^{1}$, Claudio Paoloni $^{4}$, \\ ${ }^{1}$ Ferdinand-Braun-Institut, Leibniz-Institut für Höchstfrequenztechnik, Berlin, Germany \\ ${ }^{2}$ Goethe University Frankfurt/M, Frankfurt 60323, Germany \\ ${ }^{3}$ HF Systems Engineering GmbH, Kassel 34123, Germany \\ ${ }^{4}$ Engineering Department, Lancaster University, Lancaster, United Kingdom, LA1 4YW email: c.paoloni@lancaster.ac.uk \\ ${ }^{5}$ EE Dept, University of Rome “Tor Vergata”, Rome, Italy \\ ${ }^{6}$ When Ab, Paris, France \\ ${ }^{7}$ OMMIC S.A.S., Limeil Brevannes 94453, France \\ ${ }^{8}$ Fibernova Systems, Valencia 46022, Spain \\ ${ }^{9}$ Universitat Politècnica de València, Valencia 46022, Spain
}

\begin{abstract}
The first Transmission Hub for Point to multipoint wireless distribution at D-band $(141-148.5 \mathrm{GHz})$ is presented. The paper reports the development of the main components of the transmission hub, including MMIC chipset, TWT, Image rejection filter and horn antennas.
\end{abstract}

Keywords - D-band, wireless, transmission hub, MMIC, TWT, filter, antennas.

The exploitation of the spectrum above $100 \mathrm{GHz}$ for wireless communications strongly depends on availability of affordable front end and adequate transmission power to overcome the high rain attenuation that affect most of the region of the globe. While point to point links below $90 \mathrm{GHz}$ are available in the market and have been tested up to $200 \mathrm{GHz}$ with very high gain antennas, no point to multipoint system is presently available $[1-6]$. Point to multipoint systems that are common at microwave, at the increase of the frequency are difficult to implement [7 - 10]. The use of low gain antennas for producing a wide beam needs much higher transmission power and amplification gain (not less that $15-20 \mathrm{~dB}$, assuming antennas with 15 - $25 \mathrm{dBi}$ gain for beam up to 90 degrees wide).

However, the wide available band above $100 \mathrm{GHz}$, e.g. Dband (141-174.8) has available $27 \mathrm{GHz}$, offers new opportunities for high capacity wireless transmission. The Dband allows to distribute tens gigabit per second, that can be translated in area capacity of hundreds of $\mathrm{Gb} / \mathrm{s}$ per kilometre squares. The growing traffic predictions need the deployment of high density of small cell to provide those level of data [11, 12].

The paper presents the design and development of the first Transmission Hub for Point to multipoint wireless distribution at D-band $(141-148.5 \mathrm{GHz})$. The Transmission Hub is developed in the frame of the European Commission H2020 ULTRAWAVE $[13,14]$.

\section{Electronics FOR D-BAND TRANSMISSION HuB}

\section{A. Topology}

The Transmission Hub is the core of the Point to MultiPoint D-band wireless system. It permits to distribute high data rate on a wide sectorial area illuminated by the low gain antenna. The schematic of the Transmission. Hub is shown in Fig. 1. It consists of a transmitter and receiver at D-band, two intermediate frequency levels of up-conversion and downconversion are used to connect to the modem in C-band. The first IF level is with the LO (Local Oscillator) at W-band (92 $\mathrm{GHz}$ ) to convert from D-band to Q-band. The second IF level is with the $\mathrm{LO}$ at low Q-band $(46-47.3 \mathrm{GHz})$ to convert to Cband. The D-band section includes two MMIC power amplifiers in the transmission chain. The medium power amplifier (mPA) and the Power Amplifiers (PA). It also includes a Low Noise Amplifier. The traveling wave tubes provides the transmission power for satisfying the coverage specifications [15-16].

\section{B. X4 Frequency multiplier}

A custom X4 active frequency multiplier (FM, Fig. 2) has been introduced during the system architecture assessment phase, to serve as the local oscillator signal for D-band mixers (the upconverter microphotograph is plotted in Fig.3). The frequency multiplier accepts as input a $23.3 \mathrm{GHz}$ signal and multiplies it by 4 , synthesizing a $94 \mathrm{GHz}$ signal, as required by the mixer. This is accomplished through the cascade of two $\mathrm{x} 2$ multipliers and intermediate/final amplifying buffer stages, resulting in an overall multiplier gain in the range $3-5 \mathrm{~dB}$ all over the output frequency range. Dynamic specifications require an output power level of at least $5 \mathrm{dBm}$ at $94 \mathrm{GHz}$, corresponding to a source power level of $5 \mathrm{dBm}$. The technological design process chosen for the $\mathrm{x} 4 \mathrm{FM}$ MMIC realization is the $\mathrm{D} 01 \mathrm{MH}$ provided by OMMIC. The 


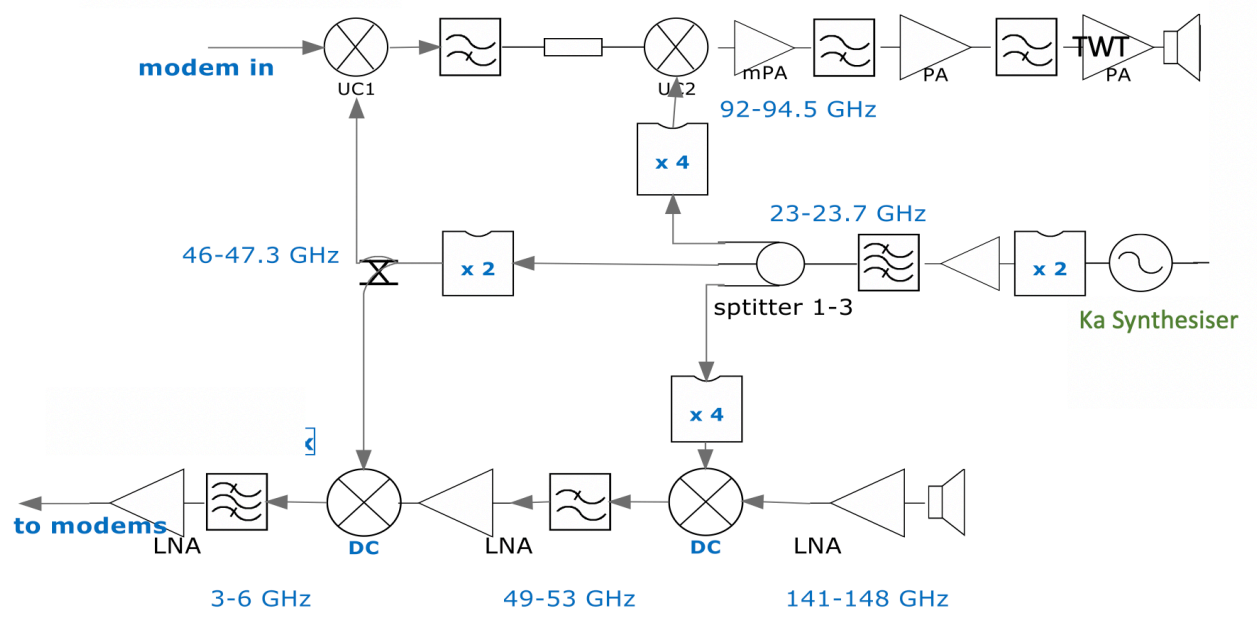

Fig. 1 Transmission Hub schematic

Table I Chipset specifications D band transceiver

\begin{tabular}{|c|c|c|c|c|c|c|}
\hline & Frequencies $\mathrm{GHz}$ & $\mathrm{G}$ (Losses) dB & P1 in $\mathrm{dBm}$ & P1out dBm & LO level dBm & S Parameters \& Isolation \\
\hline Up \& down mixer & $\begin{array}{c}\text { IF } 49 \text { - 54*; LO } 92 \text { - 95; } \\
\text { RF } 141 \text { - } 148.5\end{array}$ & -11 & -5 & & 6 & LO leakage. $<-8 \mathrm{~dB} / \mathrm{RF}$ \\
\hline Power Amplifier & $141-148.5$ & 14 & 5 & 19 & & $\mathrm{~S}_{11}, \mathrm{~S}_{22}<9 \mathrm{~dB}$ \\
\hline Medium Power A & $141-148.5$ & 10 & 5 & 10 & & $\mathrm{~S}_{11}, \mathrm{~S}_{22}<9 \mathrm{~dB}$ \\
\hline X4 Multiplier & in $23-24$; out $92-96$ & $0-1$ & 6 & 5 & 6 & $25 \mathrm{dBc} / \mathrm{f}_{0}$ \\
\hline LNA & $141-148.5$ & 20 & \multicolumn{3}{|c|}{$\mathrm{NF} 5.5 \mathrm{~dB}$} & \\
\hline
\end{tabular}

technology is a metamorphic GaAs-based and a channel length of $0.125 \mu \mathrm{m}$. The process is featured by a $f_{t}$ and a $f_{\max }$ of $150 \mathrm{GHz}$ and $250 \mathrm{GHz}$ respectively. Typical values of power density and $g_{m}$ are $300 \mathrm{~mW} / \mathrm{mm}$ and $700 \mathrm{mS} / \mathrm{mm}$ respectively. Measured performance of a typical multiplier die are plotted in Fig. 4, resulting in approximately $7 \mathrm{dBm}$ all over the output frequency range.
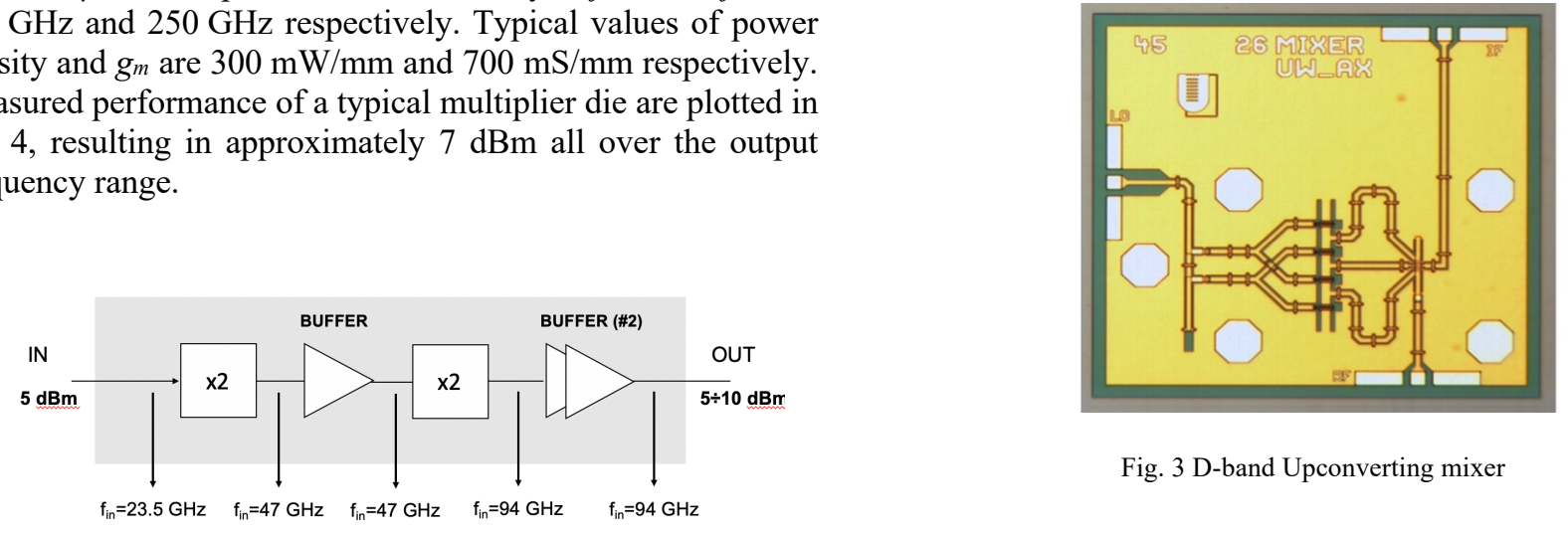

Fig. 3 D-band Upconverting mixer
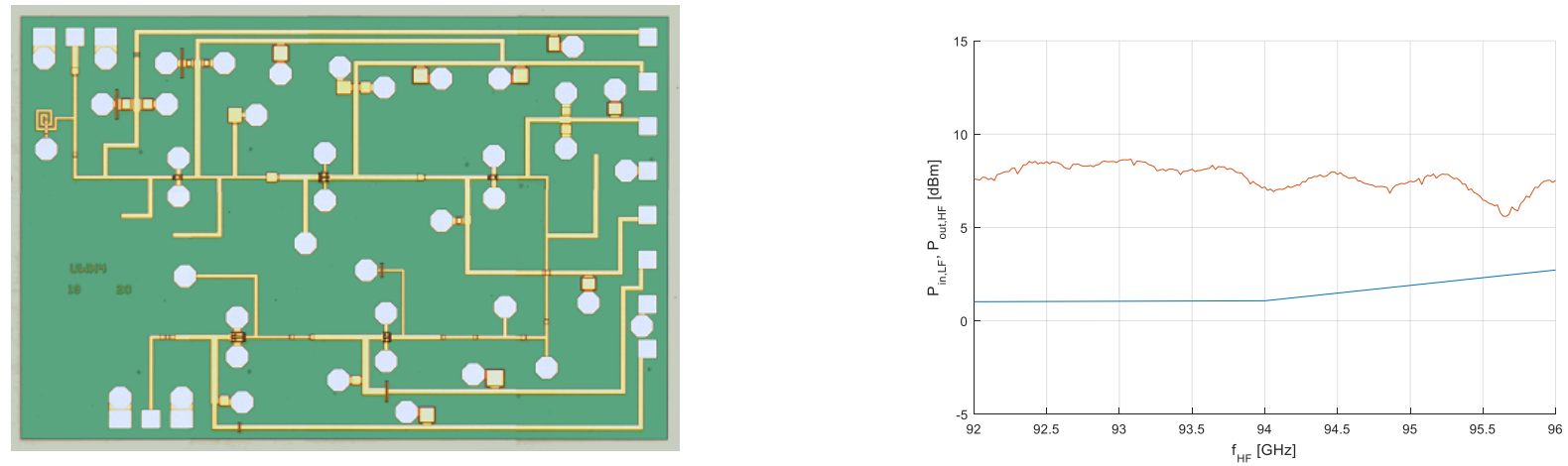

Fig. 2 X4 Frequency multiplier

Fig. 4 Power output of the X4 frequency multiplier 


\section{D-band Traveling Wave Tube}

A novel D-band TWT has been designed and is in fabrication phase. It provides $12 \mathrm{~W}$ saturated output power in the whole frequency band. All the parts where built and the final assembly is in progress. The interaction structure has been successfully measured demonstrating a wide bandwidth (Fig. 5). The TWT will provide about $12 \mathrm{~W}$ and $35 \mathrm{~dB}$ gain. The output power is about two orders of magnitude higher than the best solid-state power amplifier at the same frequency.
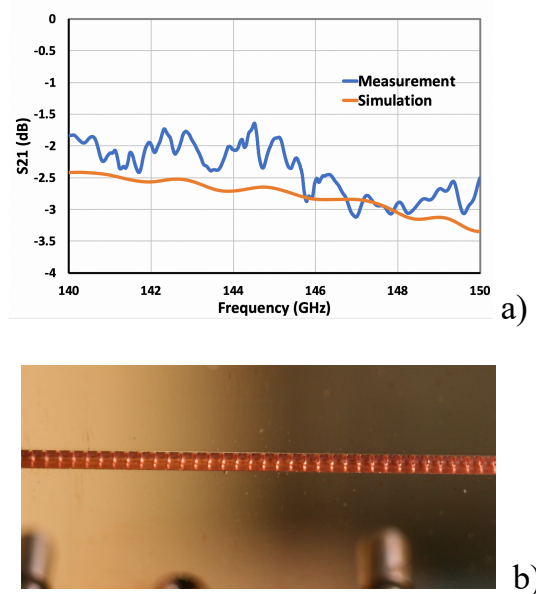

Fig. 5 D-band double corrugated waveguide a) $\mathrm{S}_{21}$ b) fabrication

\section{Low Noise Amplifier}

The Low Noise Amplifier (LNA) of the chipset, designed to operate over the 141-148 GHz band, is realised making use of the advanced OMMIC 40nm mHEMT technology [17]. The four-stage architecture is implemented in GCPW, as visible from the microphotograph in Fig. 6. Resulting measured performance include approximately $20 \mathrm{~dB}$ gain and $5.1 \mathrm{~dB}$ noise figure over the desired bandwidth.
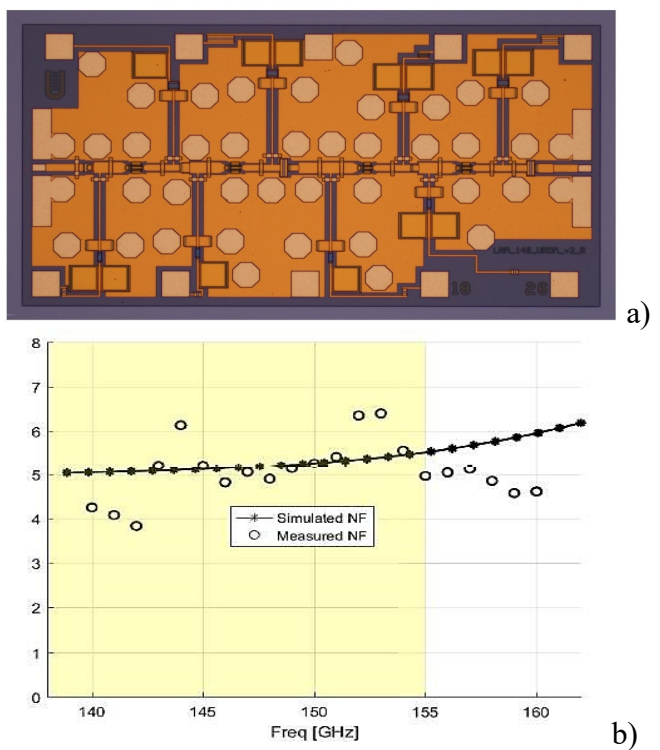

Fig. 6 a) Photograph of LNA, b) Noise Figure measurements

\section{E. D-band medium power amplifier (MPA)}

The chip photograph of the realized D-band medium power amplifier (MPA) as shown in Fig. 7. The medium power amplifier (MPA) is fabricated at the Ferdinand-Braun Institute (FBH), Berlin, in a monolithic transferred-substrate (TS) InPDHBT process. The InP transistors are transferred and bonded to a $\mathrm{Si}$ substrate in a wafer-level bonding process using benzocyclobutene (BCB). The InP DHBTs have $0.8 \mu \mathrm{m}$ wide emitters and achieve $f_{t}$ and $f_{\max }$ values of about $350 \mathrm{GHz}$ with $\mathrm{BVCEO}=4 \mathrm{~V}$. The BCB stack above the Si substrate includes three gold metal layers (G1, GD , G2) with $2 \mu \mathrm{m}, 2.5 \mu \mathrm{m}$, and $4.5 \mu \mathrm{m}$ thickness, respectively, MIM capacitors with a sheet capacitance of $0.3 \mathrm{fF} / \mu \mathrm{m}^{2}$ and $\mathrm{NiCr}$ thin film resistors (sheet resistance of $25 \Omega / \mathrm{sq}$ ). The MPA consists of a driver stage with a 2 way combined unit cell power amplifiers (PA). A unit cell power amplifier is designed of a single finger driver stage with a 2 fingers power stage. In order to achieve small signal gain more than 10, the stages are biased in class A condition.

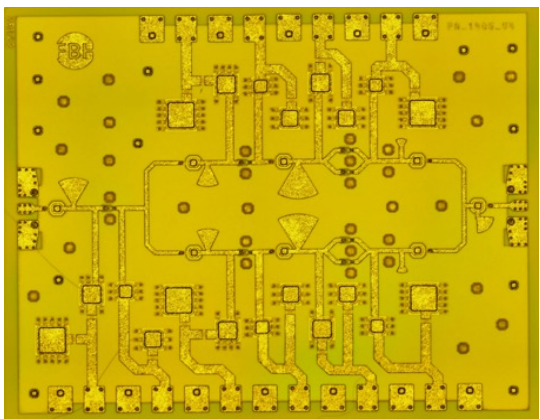

Fig. 7 The chip photograph of the D-band medium power amplifier (MPA).
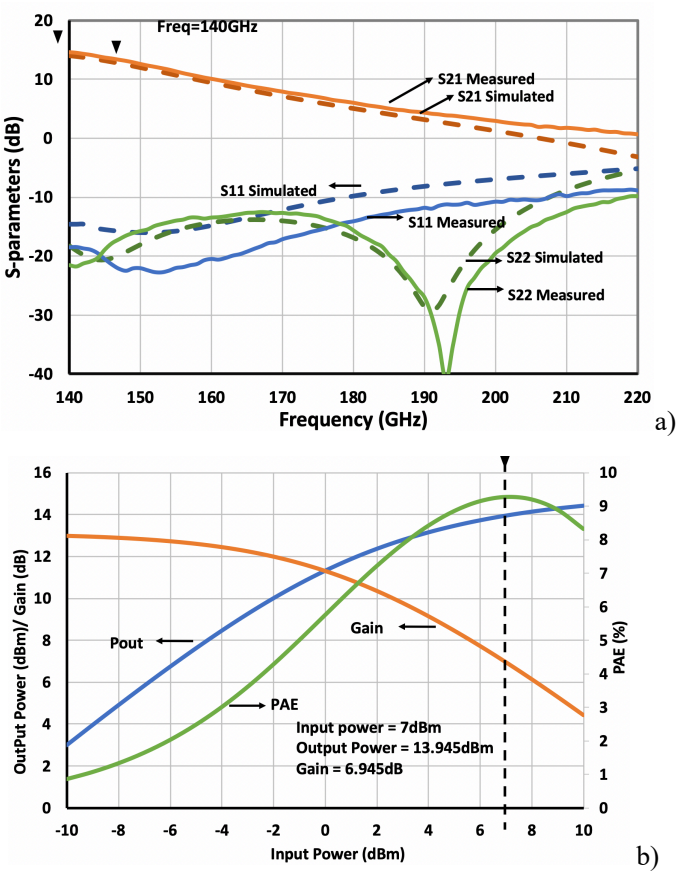

Fig. 8 Measured (solid) and simulated (dash) a) s-parameters as well as b) simulated large signal performances of the D-Band medium power amplifier (MPA). 
Fig. 8 presents the measured and simulated small signal as well as simulated large signal performances of the D-Band medium power amplifier (MPA). One can clearly see that the measured and the simulated small signal results agree well each other. The large signal measurement is ongoing, nevertheless the simulated large signal behavior predicts a P1dB compression point at an input power of $2 \mathrm{dBm}$ and at an output power of 12 $\mathrm{dBm}$. The simulated saturated output power is $14 \mathrm{dBm}$ at a maximum power-added efficiency (PAE) of $9 \%$ to be expected.

\section{F. Image Rejection filter}

The image-rejection filter is realised in waveguide technology (Fig.9a). Its high-pass characteristic is shown in Fig. 9b. The cut-off frequency is $48 \mathrm{GHz}$. The insertion loss at frequencies above $49 \mathrm{GHz}$ is less than $1 \mathrm{~dB}$. The rejection at 43 $\mathrm{GHz}$ and below is better than $50 \mathrm{~dB}$. The LO signal at 46-47 $\mathrm{GHz}$ has about $10 \mathrm{~dB}$ rejection. The rejection filter was fabricated by $\mathrm{CNC}$ milling with an insertion loss $\mathrm{IL}<1.9 \mathrm{~dB}$ at D-band.

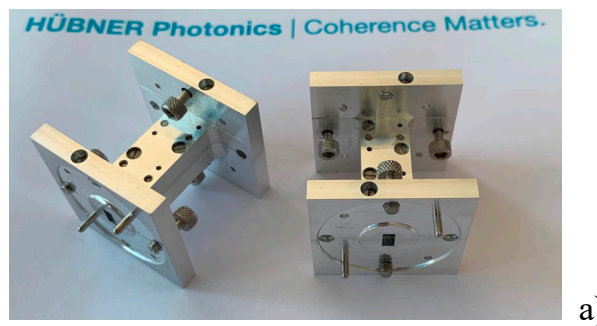

a)

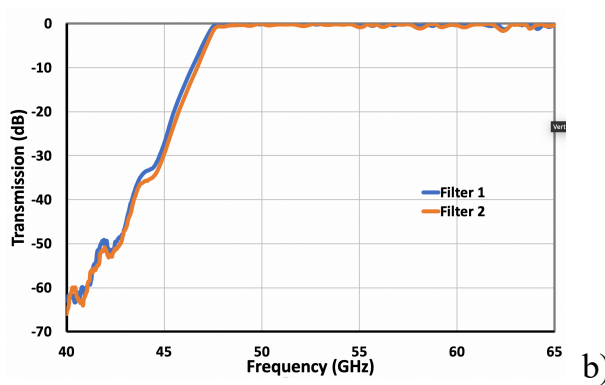

Fig. 9 Image Rejection filter, a) prototype, b) Transmission

\section{G. D-band Horn Antenna}

The transmission hub needs an antenna with a wide angular aperture to illuminate an area sector. A pyramidal horn low gain antenna was designed by the electromagnetic simulator CST, fabricated (Fig. 10a) and then measured in an anechoic chamber.

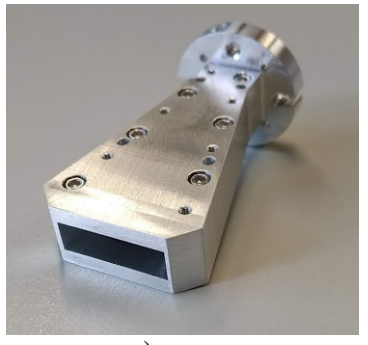

a)

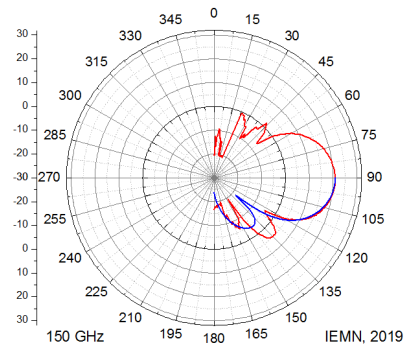

b)
The antenna was designed to have a gain of about $20 \mathrm{~dB}$ and main lobe width of $30^{\circ}$ in the frequency range $140-150 \mathrm{GHz}$. The comparison of the measured and simulated radiation pattern at $150 \mathrm{GHz}$ is reported in Fig. 10b.

\section{CONCLUSION}

The D-band transmission hub is in advanced fabrication stage. The novel components at D-band for the system have been described. Most of the components are at the state of the art. The full system will be assembled and tested in real environment.

\section{ACKNOWLEDGMENT}

The work has received funding from the European Union's Horizon 2020 research and innovation programs under grant agreement no 762119. This work reflects only the author view's and the Commission is not responsible of any use that may be made of the information it contains

\section{REFERENCES}

[1] Dhillon, S. S. et. al, "The 2017 terahertz science and technology roadmap", J. Phys. D: Appl. Phys. 2017, 50, 043001.

[2] T. Nagatsuma et al. Advances in terahertz communications accelerated by photonics, Nature Photonics 10, 371-379 (2016)

[3] Z. Pi and F. Khan, "An introduction to millimeter-wave mobile broadband systems," IEEE Comm. Magazine, pp. 101-107, June 2011.

[4] X. Li, et al. , "Fiber-wireless- fiber link for 100-Gb/s PDM-QPSK signal transmission at W-band,” IEEE Photon. Technol. Lett., Jul. 2014.

[5] T.S. Rappaport et al., “ Millimeter Wave Mobile Communications for 5G Cellular: It will work!”, IEEE Access, pp. 335-349, Mai 2013.

[6] J. Takeuchi, et. al. "10-Gbit/s Bi-directional wireless data transmission system using 120-GHz-band ortho-mode transducers," 2012 IEEE Radio and Wireless Symposium, 2012, pp. 63-66.A.

[7] R. Taori and A. Sridharan. "Point-to-multipoint in-band mmwave backhaul for 5G networks,", IEEE Communications Mag., pp.195-201, January 2015.

[8] F. Magne, A. Ramirez, C. Paoloni, "Millimeter Wave Point to Multipoint for Affordable High Capacity Backhaul of Dense Cell Networks", IEEE Wireless Communications and Networking Conference 2018, WCNC 2018, Barcelona, Spain, April 2018.

[9] J. Shi, L. Lv, Q. Ni, H. Pervaiz and C. Paoloni, "Modeling and Analysis of Point-to-Multipoint Millimeter Wave Backhaul Networks," in IEEE Trans. on Wireless Communications, vol. 18, no. 1, pp. 268-285, Jan. 2019

[10] C. Paoloni et al., "Transmisson Hub and Terminals for Point to Multipoint W-Band Tweether System," 2018 European Conference on Networks and Communications (EuCNC), Ljubljana, Slovenia, 2018.

[11] Ericsson Mobility Report, November 2018 on line https://www.ericsson.com/en/mobility-report

[12] Cisco Visual Networking Index: Global Mobile Data Traffic Forecast Update, 2016-2021 White Paper, Cisco Mobile VNI, 2017

[13] ULTRAWAVE website [Online]. Available: http://ultrawave2020.eu

[14] C. Paoloni et al., "Technology for D-band/G-band ultra capacity layer," 2019 European Conference on Networks and Communications (EuCNC), Valencia, Spain, 2019, pp. 209-213, doi: 10.1109/EuCNC.2019.8801983.

[15] R.Basu, L. R. Billa, R. Letizia, C. Paoloni, "Design of sub-THz traveling wave tubes for high data rate long range wireless links", 2018 Semicond. Sci. Technol. 33124009

[16] R.Basu, L. R. Billa, R. Letizia, C. Paoloni, "Design of D-band Double Corrugated Waveguide TWT for Wireless Communications “, Proc. IEEE 20th Int. Vac. Electron. Conf., Busan, South Kores, April. 2019.

[17] R. Cleriti et al., "D-band LNA using a 40-nm GaAs mHEMT technology," 2017 12th European Microwave Integrated Circuits Conference (EuMIC), Nuremberg, 2017, pp. 105-108, doi: 10.23919/EuMIC.2017.82306

Fig. 10 a) D-band horn antenna and b) radiation diagram 